\title{
Balancing size and density segregation in bidisperse dense granular flows
}

\author{
Deepak R. Tunuguntla ${ }^{1, \star}$ and Anthony R. Thornton ${ }^{1, \star \star}$ \\ ${ }^{1}$ Multi-Scale Mechanics Group, Dept. of Thermal and Fluid Engineering, University of Twente, Enschede, The Netherlands.
}

\begin{abstract}
Several experimental studies have illustrated a balance between the segregation forces arising due to size- and density-differences. However, no detailed studies have been carried out to quantify this balance. In 2014, by utilising discrete particle simulations, we presented a simple relationship between the particle sizeand density-ratio, $\hat{s}^{a}=\hat{\rho}$, where ' $a$ ' determines whether the partial pressure scales with the diameter, surface area or volume of the particle. For a 50:50 mix (in volume) of bidisperse granular mixtures, we found the partial pressure to scale with the volume of the particle, i.e. $a=3$. Moreover, there also exists a range of size- and density-ratios that satisfy the relation $\hat{s}^{3}=\hat{\rho}$, where the bidisperse mixture remains homogeneously mixed. However, in this proceeding, we deviate from the conventional 50:50 mixes and consider a slightly extreme case of mixes, such as the 10:90 (in volume) mixes, which are often found in nature and industries. By doing so we observe that the partial pressure does not scale with the particle volume and, more importantly, the zerosegregation relation is not as simple as $\hat{s}^{a}=\hat{\rho}$. However, there does exist a range of size- and density-ratios for which the mixture weakly segregates.
\end{abstract}

\section{Introduction}

Granular mixtures often tend to arrange themselves in certain patterns when stirred, shaken or sheared [1]. These mixtures often comprise of constituents varying in size, density, inelasticity, shape, surface roughness etc. When such polydisperse mixtures are subjected to external forces, individual studies [2-10] confirm the influence of these constituent properties in forming patterns, e.g., segregation or de-mixing. However, in free-surface flows over inclined channels, differences in size and density are the primary factors for segregation.

Among several mechanisms responsible for segregation, kinetic sieving [11] is the dominant one in dense granular flows. Kinetic sieving is a trivial mechanism. As the mixture flows down the inclined channel, fluctuations in the local pore space cause smaller particles to fall into space/gaps created beneath them. The fines, i.e. small sized particles, easily fit into these pores leading to gradual percolation of them towards the base of the flow. Simultaneously, force imbalances lever/squeeze the coarser particles towards the surface. This simple mechanism results in stratified layers which one terms as segregation. Opposing kinetic sieving is diffusive remixing which causes random motions of particles as they collide and shear over each other [12]. Based on the relative strength of either mechanisms, the mixture strongly or weakly segregates. Apart from kinetic sieving, which is a purely size-based effect, buoyancy effects due to differences in particle density also play a major role in particle segregation [13]. For

\footnotetext{
^e-mail: d.r.tunuguntla@utwente.nl

${ }^{\star}$ e-mail: a.r.thornton@utwente.nl
}

bi-disperse mixtures, varying in particle size and density, experiments [14] and simulations [6] indicate a balance between the two driving mechanisms, i.e between kinetic sieving and buoyancy, which in turn keeps the mixture homogeneously mixed.

Although experiments do provide authentic information, current state-of-the-art experimental techniques are still hampered due to some inherent constraints. Thereby, as an alternative to experiments, we utilise informationrich discrete particle simulations (DPMs) [15]. Given $\hat{s}$ and $\hat{\rho}$ is denoted as size- and density-ratio for a bidisperse mixture, in 2014, Tunuguntla et al. [6] proposed a DPMs validated theoretical relation

$$
\hat{s}^{a}=\hat{\rho},
$$

where ' $a$ ' here determines whether the partial pressure scales with the diameter, surface area or volume of the particle $^{1}$. More importantly, the relation further indicates that there exists a possible range of size- and density-ratios for which the bidisperse mixture very weakly or does not segregate. Although, the above relation has been validated using DPMs, the simulation setup in [6] considered a conventional shallow 50:50 bidisperse mixture alone. By 50:50 we imply that each particle type occupies $50 \%$ of the total solid volume. Nevertheless, for a given range of sizeand density-ratios, they found the exponent $a=3$ implying that the partial pressure scales with the volume of the particle.

In this proceeding, by utilising the discrete particle simulations as setup in Sec. 2, we aim to scrutinise the

\footnotetext{
${ }^{1}$ For more details concerning the definition of partial pressures, see Tunuguntla et al. [6].
} 


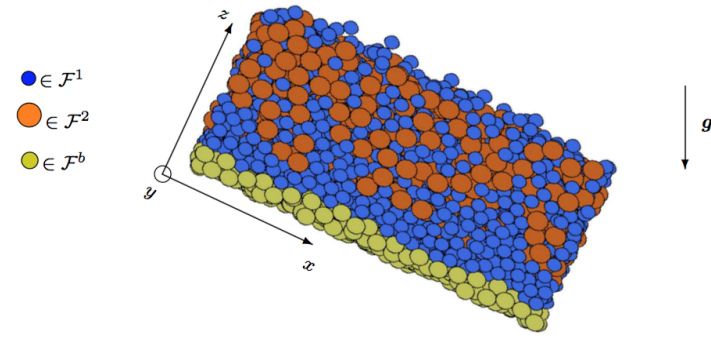

Figure 1. A illustration of a bidisperse mixture flowing in a periodic box inclined at $26^{\circ}$ to the horizontal (discrete particle simulation), adapted from Tunuguntla et al. [16]. Colours/shades indicate the base/boundary (yellowish green, $\mathcal{F}^{b}$ ), species type-1 and type-2 (blue, $\mathcal{F}^{1}$ and red, $\mathcal{F}^{2}$ ). We define the bulk as $\mathcal{F}^{1} \cup \mathcal{F}^{2}$.

generality of the above relation (1). In order to do so, we sway away from the conventional 50:50 bidisperse mixtures and consider a 10:90 bidisperse mixtures, i.e. one of the particle type occupies $10 \%$ of the total solid volume in comparison to the other particle type. Moreover, it is the 10:90 mixes that are more common, in many natural and industrial scenarios, than the conventional 50:50 mixes. For example, in a pharmaceutical industry the final chemical composition of a drug often contains larger amounts of excipient than the active ingredient, and practically all marketed drugs contains excipients ${ }^{2}$. Thereby resulting in dilute granular mixtures such as the 10:90 bidisperse mixtures. When these dilute mixtures are subjected to external shear while transporting and processing, phenomena such as particle segregation often lead to unwanted inhomogeneities in the mixtures, which in turn hamper the final drug composition. Hence, demanding for thorough investigations concerning particle segregation in dilute mixtures. Here, we consider dilute bidisperse granular flows in inclined channels, which is a commonly utilised method of transport in industry.

\section{Simulation setup}

For a range of size- and density-ratios, $\hat{s} \in[0.3,1.4]$ and $\hat{\rho} \in[0.3,1.4]$, fully three-dimensional (3D) discrete particle simulations (DPM) are set up in our in-house opensource particle solver, MercuryDPM [17].

A cuboidal box, periodic in $x$ - and $y$-direction, is inclined to the horizontal at $26^{\circ}$, with the dimensions $L \times W \times H=20 d_{m} \times 10 d_{m} \times 10 d_{m}$. Here, $d_{m}$ represents the mean particle diameter defined as $d_{m}=\phi^{1} d_{1}+\phi^{2} d_{2}$, where $\phi^{i}$ and $d_{i}$ denotes the solid volume fraction and diameter of type-i particle species. In this proceeding, we define the particle size- and density-ratio as $\hat{s}:=d_{2} / d_{1}$ and $\hat{\rho}:=\rho_{p}^{2} / \rho_{p}^{1}$, where $\rho_{p}^{i}$ is defined as the particle density of particle type- $i$.

In order to add a rough base (bottom) to the cuboidal box, we fill the periodic box with a randomly distributed

\footnotetext{
${ }^{2}$ By excipients, we mean bulking agents or fillers or diluents which facilitate drug solubility and easy dosage whereas the active ingredient is the one responsible for killing the virus for example.
}

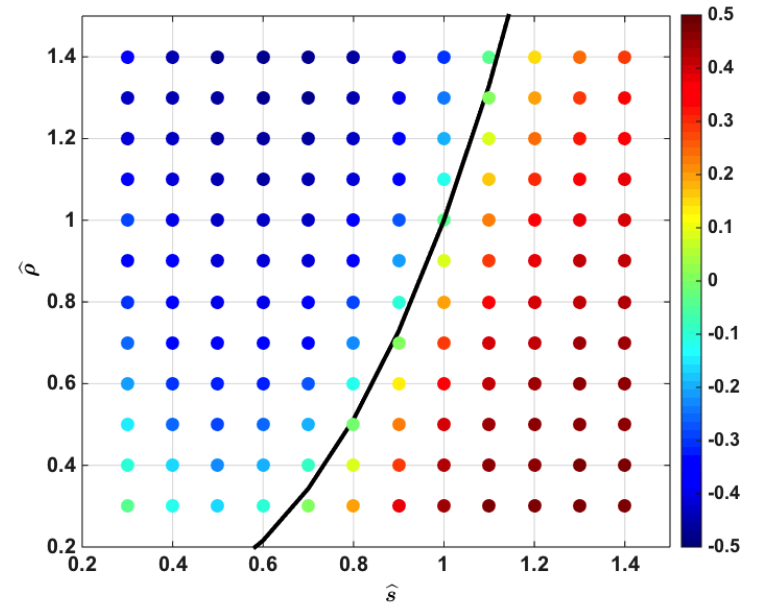

Figure 2. For a 50:50 initially homogeneously mixed bidisperse mixture, we plot $\widehat{D}_{\text {com }}=\left(\hat{z}_{\text {com }}^{2}-\hat{z}_{\text {com }}^{\text {bulk }}\right) / \hat{z}_{\text {com }}^{\text {bulk }}$ for different values of $\hat{s}$ and $\hat{\rho}$. The black solid line represents the weak segregation line, which satisfies the simple relation in Eqn. 1 for $a=3$. Note that the above plot is adapted from Tunuguntla et al. [6].

set of particles with uniform diameter $d_{m}$. Once filled, they are simulated until a static layer of about 12 particles thickness is produced. To form a rough base, a slice of particles with centres between $z \in[9.3,11] d_{m}$ is taken and translated 11 mean particle diameters downwards, see Weinhart et al. [18]. Additionally, to make sure no particles fall through the created rough base, a solid flat wall is placed underneath this static layer. After the creation of a rough base, the box is then filled with a homogeneously mixed bidisperse mixture with a specific size- and density-ratio, as seen in Fig. 1. For more details regarding the procedure for determining the number of particles and the contact model, please see Tunuguntla et al. [16, 19].

Given the particle size- and density-ratio, $\hat{s} \in[0.3,1.4]$ and $\hat{\rho} \in[0.3,1.4]$, total number of particles, $N_{1}$ and $N_{2}$, and contact model parameters, the particles are randomly positioned into the box with dimensions $\widehat{V}_{b o x}=20 \times 10 \times H$ where $\mathrm{H}$ is defined as $\left(N_{1}+N_{2}\right) / 300$. However, when an inserted particle at any position overlaps with another particle, the insertion is rejected and the insertion domain is enlarged by increasing $H$ to $H+0.01 H$ to ensure that there is enough volume in the box to accomodate all the particles. Thus, leading to an initially loosely packed mixture. As the simulation commences, the particles compact enough to reach a flow depth of $12 d_{m}$, see Fig. 1, giving the particles enough energy to initialise flow. For more details see $[16,18,19]$.

With the particle simulations setup in the above elucidated manner, the following section presents our observations.

\section{Results}

Homogeneously mixed bidisperse mixtures are simulated for a range of particle size- and density-ratios, $\hat{s} \in$ $[0.3,1.4]$ and $\hat{\rho} \in[0.3,1.4]$, till a steady state is reached. 


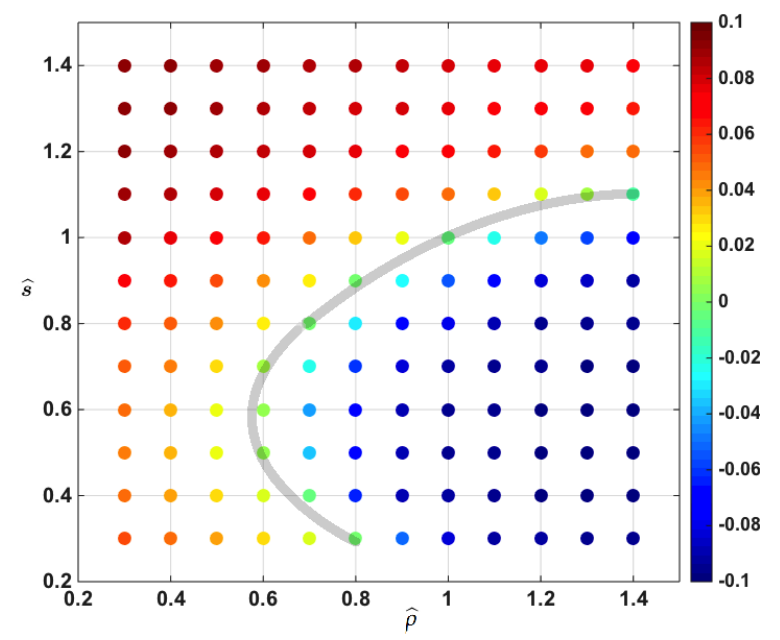

Figure 3. For a 10:90 initially homogeneously mixed bidisperse mixture, we plot $\widehat{D}_{\text {com }}=\left(\hat{z}_{\text {com }}^{2}-\hat{z}_{\text {com }}^{\text {bulk }}\right) / \hat{z}_{\text {com }}^{\text {bulk }}$ for different values of size- and density-ratios, $\hat{s}$ and $\hat{\rho}$. Similar to the black solid line in Figure 2, the greyish transluscent line in the above plot approximately represents the weak segregation line in 10:90 dilute mixtures. However, this segregation line is no longer captured by the simple relation stated in Eqn. 1. Hence, implying that the value of $a$ is dependent on the mixture volume fraction. Also note that the $x$ - and $y$-axis labels are flipped when compared to the ones in Fig. 2.

To define a steady mixture state, we track the vertical center of mass $\left(\hat{z}_{\text {com }}\right)$ of both the type- 1 and -2 species as a function of time, similar to the plots illustrated in Fig. 3 of Tunuguntla et al. [6]. As $\hat{z}_{\text {com }}$ reaches a steady value in time, we define our mixture to have reached a steady state. With this at hand, we first compute the relative difference between the vertical center of mass of the type- 2 species $\left(\hat{z}_{\text {com }}^{2}\right)$ and the bulk $\left(\hat{z}_{\text {com }}^{\text {bulk }}\right)$ and then normalise it with the bulk's vertical center of mass, $\hat{z}_{\text {com }}^{\text {bulk }}$. By this, we arrive at a normalised relative measure $\widehat{D}_{\text {com }}=\left(\hat{z}_{\text {com }}^{2}-\hat{z}_{\text {com }}^{\text {bulk }}\right) / \hat{z}_{\text {com }}^{\text {bulk }}$ as a function of $\hat{s}$ and $\hat{\rho}$.

As a brief recap, for 50:50 mixes Tunuguntla et al. [6] illustrated the normalised relative measure, $\widehat{D}_{\text {com }}$, for type2 particle species, which is also shown in Fig. 2. Given the flows are in a steady state, for $\widehat{D}_{\text {com }}>0$, type- 2 particle species appear to be near the free surface of the flow; whereas for $\widehat{D}_{\text {com }}<0$, type- 2 particle species appear to be near the base of the flow. Moreover, Fig. 2 also illustrates the important aspect of very weak/zero segregation, which mainly occurs due to a balance between the segregation forces arising due to size- and density-differences. Additionally, the black solid line denotes the weak/zero segregation line which is represented by the relation $\hat{s}^{a=3}=\hat{\rho}$. Thus, also implying that the partial pressure is scaled by the volume of the particle, as $a=3$.

On the other hand, to verify the generality of the relation $\hat{s}^{a}=\hat{\rho}$, we further simulated 10:90 mix of homogeneously mixed bidisperse mixtures, for the same earlier mentioned range of size- and density-ratios, $\hat{s} \in[0.3,1.4]$ and $\hat{\rho} \in[0.3,1.4]$. Once again, in Fig. 3, we plot the normalised relative measure, $\widehat{D}_{\text {com }}$, for type- 2 particle species.
As illustrated in Fig. 3, similar to 50:50 mixes, there also exists a range of $\hat{s}$ and $\hat{\rho}$ for which the mixture exhibits weak or zero-segregation. However, the zero-segregation line is no longer a simple relation between the size- and density-ratio, as shown in Eqn. 1. There definitely exists a deviation from the relation stated in (1), implying that the relation $\hat{s}^{a=3}=\hat{\rho}$ cannot be generalised to dilute mixtures. Additionally, given the relation in (1) is not satisfied for $a \neq 3$, the partial pressure does not scale with the volume of the particle, as observed for mixtures with 50:50 volume ratio. Nevertheless, on closer observation of Fig. 3, we do also see that for $\hat{\rho} \in[0.6,0.8]$ there exists a set of size-ratios for which the mixture remains homogeneously mixed. For example, for the same density-ratio, $\hat{\rho}=0.7$, there are two size-ratios $\hat{s}=0.4$ and $\hat{s}=0.8$ at which the mixture weakly segregates. Thus, asking for a detailed future investigation.

\section{Conclusions}

By utilising discrete particle simulations, for both 50:50 and 10:90 mixes, we show that there exists a range of particle size- and density-ratios for which a homogeneously bidisperse, in size and density, mixture demonstrates weak/zero segregation. However, the simple relation (1) that captures the zero-segregation line for 50:50 mixes, does not apply for 10:90 mixes, implying that the relationship cannot be generalised to general mixes. Moreover, the exponent $a$ in the relation (1) could be considered as a material parameter which is a function of particle volume fraction or material properties. However, further detailed study is required for investigating the segregation dynamics due to size- and density-differences.

\subsection{Future outlook}

Understanding particle segregation due to size- and density-differences is vital due to its wide range of applications in both nature and industry. Although, discrete particle simulations give detailed insight into the segregation dynamics, modelling realistic industrial flows is computationally expensive. Thereby, continuum models appear to be an efficient alternative. Similar to the several models developed to model size-based particle segregation models [19], developing efficient segregation models to account for, both, size- and density-differences would be useful. Although there exist a few models that account for these two effects [6, 20,21], a detailed study using experiments or simulations would still be beneficial for better understanding and development of efficient continuum models.

\section{Acknowledgements}

The authors would like to thank the Dutch Technology Foundation STW for its financial support and user committee meetings of STW-Vidi project 13472, Shaping Segregation: Advanced Modelling of Segregation and its Application to Industrial Processes. 


\section{References}

[1] J. Duran, Sands, Powders, and Grains (Springer, 2000)

[2] S. Wiederseiner, N. Andreini, G. Épely-Chauvin, G. Moser, M. Monnereau, J.M.N.T. Gray, C. Ancey, Phys. Fluids 23, 013301 (2011)

[3] K. van der Vaart, P. Gajjar, G. Epely-Chauvin, N. Andreini, J.M.N.T. Gray, C. Ancey, Phys. Rev. Lett. 114, 238001 (2015)

[4] F. Guillard, Y. Forterre, O. Pouliquen, J. Fluid Mech. 807 (2016)

[5] A. Tripathi, D.V. Khakhar, J. Fluid Mech. 717, 643 (2013)

[6] D.R. Tunuguntla, O. Bokhove, A.R. Thornton, J. Fluid Mech. 749, 99 (2014)

[7] H. Xiao, P.B. Umbanhowar, J.M. Ottino, R.M. Lueptow, Modelling density segregation in flowing bidisperse granular materials, in Proc. R. Soc. A (The Royal Society, 2016), Vol. 472, p. 20150856

[8] R. Brito, R. Soto, Eur. Phys. J. Special Topics 179, 207 (2009)

[9] B.L. Pollard, H. Henein, Can. Metall. Q. 28, 29 (1989)

[10] S. Ulrich, M. Schröter, H.L. Swinney, Phys. Rev. E 76, 042301 (2007)
[11] J.A. Drahun, J. Bridgwater, Powder Technol. 36, 39 (1983)

[12] S.S. Hsiau, M.L. Hunt, J. Fluid Mech. 251, 299 (1993)

[13] D.V. Khakhar, J.J. McCarthy, J.M. Ottino, Chaos 9, 594 (1999)

[14] G. Felix, N. Thomas, Phys. Rev. E 70, 051307 (2004)

[15] S. Luding, European Journal of Environmental and Civil Engineering 12, 785 (2008)

[16] D.R. Tunuguntla, A.R. Thornton, T. Weinhart, Comput. Part. Mech. 3, 349 (2016)

[17] A.R. Thornton, D. Krijsgman, R. Fransen, S. Gonzalez, D.R. Tunuguntla, A. te Voortwis, S. Luding, O. Bokhove, T. Weinhart, Newsletter Enginesoft 1, 48 (2013)

[18] T. Weinhart, A.R. Thornton, S. Luding, O. Bokhove, Granul. Matt. 14, 531 (2012)

[19] D.R. Tunuguntla, T. Weinhart, A.R. Thornton, Comput. Part. Mech., DOI: 10.1007/s40571-016-0136-1 (2016)

[20] B. Marks, P. Rognon, I. Einav, J. Fluid Mech. 690, 499 (2012)

[21] J. Gray, C. Ancey, Journal of Fluid Mechanics 779, 622 (2015) 